\section{Differential drug resistance to anti- tumour necrosis factor agents in rheumatoid arthritis}

\section{P I Sidiropoulos, D T Boumpas}

\section{A significant number of patients receiving infliximab require dosing adjustments while some patients may indeed develop drug resistance}

$\mathrm{R}$ heumatoid arthritis (RA) is a chronic, inflammatory autoimmune disease that leads to progressive joint destruction and disability. New biological drugs have been added to our armamentarium since 1999, including agents targeting tumour necrosis factor $\alpha(\mathrm{TNF} \alpha)$. Among them, infliximab, etanercept, and adalimumab in randomised controlled trials (RCTs), reduced signs and symptoms of RA and protected joints from structural damage more effectively than conventional disease modifying antirheumatic drugs (DMARDs). ${ }^{1-3}$ However, residual disease activity remains a considerable problem and remissions are seen in $<30 \%$ of patients with established disease. ${ }^{4}$

To date, head-to-head comparisons among anti-TNF $\alpha$ agents do not exist, in part because of the large sample size required to demonstrate either differences between, or equivalence of, treatments. In the absence of head-to-head randomised comparisons of anti-TNF $\alpha$ agents, claims that one drug is better than the others have been met with scepticism by practising physicians They generally view these drugs as a class of agents with comparable efficacy and toxicity, the final choice of which is left to patient preference and the physician's experience with each of them.

RCTs are indispensable in determining clinical efficacy of new treatments, but their ability to predict long term outcomes, side effects, and their wider applicability in a general population is limited. Long term observational studies (LOS) and patient registries provide additional information, because there are no exclusions in enrolment and they maintain long term follow up. Recently, three European registries-in Switzerland, southern Sweden, and Germany-have attempted to explore the comparative efficacy and tolerability of available anti-TNF agents. ${ }^{5-7}$

In this editorial, we examine drug effectiveness and resistance to anti-TNF $\alpha$ agents based on data from these registries. For the purposes of this discussion we define drug resistance as secondary failure-that is, loss of a previously satisfactory therapeutic response to the anti-TNF $\alpha$ agents.

\section{BIOLOGICAL THERAPY REGISTRIES IN RA: INITIAL DATA}

In the Swiss registry of patients with RA receiving biological treatments, clinical information is updated every time a patient's disease status necessitates a change in their antirheumatic treatment. Most importantly, patients originate from a range of clinical settings, with $40 \%$ coming from private practices, $30 \%$ from non-academic centres, and $30 \%$ from academic centres.

\section{"The efficacy of infliximab at recom- mended low dose, but not etaner- cept, appears to decrease over time"}

A group of investigators led by Axel Finckh has taken advantage of this cohort to explore the relative effectiveness of biological treatments in inhibiting radiological progression. ${ }^{8}$ In this issue of the Annals, the evidence for differential acquired resistance to the three anti-TNF $\alpha$ agents in common use today-namely, infliximab, etanercept and adalimumab, is examined. ${ }^{5}$ Using this cohort of 1198 patients and with a respectable 1450 patient-years of anti$\mathrm{TNF} \alpha$ treatment, they found that the rate of intensification of traditional treatment with DMARDs over time (a proxy for decreased efficacy) was significantly higher for infliximab than with other two agents. An average dose increase of $+12 \%$ was found for infliximab after 1 year and $18 \%$ after 2 years, but no difference in discontinuation rates among the agents was found. The investigators found a lower therapeutic response to infliximab than with etanercept after the first 6 months of treatment, although someone may question the clinical significance of the observed differences (a difference of 0.27 in the DAS28 between treatments). After 1 year of treatment about half of the patients in the registry had a good to moderate response by EULAR response criteria.

The authors have used a composite definition for "drug resistance", with the intensification of DMARD co-therapy, gradual dose escalation of biological agents, and drug discontinuation rates as proxies. It is not clear from their analysis whether dose escalation reflects inadequate dosing or secondary failure, which in our opinion may be a better proxy for drug resistance. The finding that dose escalation was steepest during the first year and slowed down during the second year suggests that although both mechanisms may be implicated, inadequate dosing may have been the main mechanism involved.

In a similar study based on the South Swedish Arthritis Treatment Group registry, Kristensen et al introduced a new index of drug efficacy in clinical practice (the LUNDEX index) that takes into account both drug efficacy and tolerability over time. ${ }^{6}$ They compared etanercept with infliximab, and found that etanercept had higher LUNDEX values, mostly because of higher rates of adherence to treatment than with infliximab. In that study, the rate of discontinuation for lack of efficacy for etanercept was about $15 \%$ at 3 years while for infliximab it was about 20\% $(\mathrm{p}=0.018)$.

Finally, in the German registry the rates of discontinuation of biological therapy were about $20 \%$ at 1 year for both etanercept and infliximab. ${ }^{7}$ These figures although higher that those reported in RCTs were significantly better than those reported for DMARDS (about 49\% after adjustments for disease status at study entry).

LOS are plagued by generic problems, including missing data, generalisability, repeated measures on the same person, measures taken at varying time points from symptom onset, patient recruitment/selection bias, and non-random assignment to treatments. Thus, in general, LOS cannot usually be used to detect differences between interventions. ${ }^{9}$ Despite their limitations, these studies, provide useful, although not definitive, data on the comparison among biological agents in a "real life" situation.

Overall, these studies suggest that all three biological agents have an excellent drug survival, while discontinuations due to treatment failure are roughly the same across the three registries. When etanercept and infliximab are compared, no consistent differences are 
found across these registries. Of note, treatment failure decisions were not based on a predefined level of disease activity but on the clinical judgment of treating physicians. Certainly the data from the Swiss registry raise the possibility of drug resistance to anti-TNFa agents, especially to infliximab, but the extent of this phenomenon cannot be adequately determined.

\section{IS DRUG RESISTANCE UNIQUE TO ANTI-TNF AGENTS?}

RA treatment is instituted with established DMARDs given either as sequential monotherapy or in a variety of combinations. Dose adjustments are often required to keep disease under control, with as many as two thirds of patients requiring dose adjustment of conventional DMARDs in the TICORA study. ${ }^{10}$ Clinical experience suggests that reactivation of inflammation often occurs despite continuous drug treatment, indicating that the efficacy of DMARDs may be compromised with long term treatment. It is not known whether this is due to progression of the disease (associated with a change in the relative importance of the pathogenetic mechanisms involved) or to the development of cellular drug resistance to DMARDs. The molecular basis of failure of DMARD treatment has been examined in a limited number of small scale clinical studies. Most studies focused mainly on the multidrug resistance proteins, like $\mathrm{P}$-glycoprotein (P-GP or $A B C B 1)$, while more recent studies have explored multidrug resistanceassociated proteins (MRP)l, a multidrug efflux pump. ${ }^{11}$ Cross resistance to multiple DMARDs, including corticosteroids, may be mediated by MRPl. The clinical significant of this resistance needs to be explored further.

\section{DRUG RESISTANCE OR INADEQUATE DOSING?}

Initial data from LOS while confirming the safety, efficacy, and tolerability of the new drugs first shown in RCTs, have demonstrated variable rates of treatment discontinuation (from 5\% up to $20 \%$ ) due to inefficacy. ${ }^{6}{ }^{12}$ Furthermore, many investigators have reported that dose adjustments are necessary for a significant number $(60$ $70 \%$ ) of patients receiving infliximab. ${ }^{4}{ }^{12}{ }^{13}$ Whether this is due to inadequate dosing or secondary failures is difficult to discern from these studies. van Vollenhoven et al, in a study thus far reported only in abstract form, found a yearly rate of secondary loss to infliximab of about $5 \%{ }^{14}$; about $20 \%$ of all discontinuations were attributed to secondary loss of efficacy. ${ }^{15}$
It is important to know whether dose escalation represents true drug resistance or is due to suboptimal initial drug dosing. Although, the first year data from the ATTRACT trial suggested that the ACR20 response rates were not significantly different among the infliximab treatment groups, subjects receiving $3 \mathrm{mg} / \mathrm{kg}$ infliximab every 8 weeks had a significantly lower ACR50 response rate than subjects in the three higher dosage groups after 54 weeks of treatment. Moreover, after week 30, the proportion of ACR20 and ACR50 responders waned in the group receiving $3 \mathrm{mg} / \mathrm{kg}$ every 8 weeks, which contrasted with the relatively constant rates of response of the other three infliximab treatment groups over time. ${ }^{1}$ Pharmacokinetic studies have shown that with the $3 \mathrm{mg} / \mathrm{kg}$ infliximab dose, $22-30 \%$ of the subjects have levels below the quantification limits at the end of the dosing period, during which these patients may not be covered. ${ }^{16}$ This compares with mean (SD) minimum steady state concentrations for adalimumab of 3.8 (2.1) mg/l (at a dose of $40 \mathrm{mg}$ every other week), while the EC50 (effective concentration that leads to $50 \%$ of the observed effect) for the swollen and tender joint counts is estimated at $1.35(0.43) \mathrm{mg} / \mathrm{l}^{17}$

\section{About 1 in 5 biological drug dis- continuations may be due to a secondary loss of efficacy"}

In summary, these data from the ATTRACT trial provide initial evidence that clinical improvement from infliximab treatment is dose dependent and that the $3 \mathrm{mg} / \mathrm{kg}$ infliximab dose may be subtherapeutic in a proportion of patients at the end of the 8 week dosing period. Clinical experience also supports this impression; in our experience interval adjustment is necessary in about two thirds of all patients. ${ }^{4}$ Stern and Wolfe also reported that the mean dose for infliximab was increased from $3.6 \mathrm{mg} /$ $\mathrm{kg}$ at baseline to about $4.9 \mathrm{mg} / \mathrm{kg}$ at 1 year and stabilised at 5.1 at 2 years. ${ }^{12}$ In another LOS the same authors reported a mean dose at 18 months of $5 \mathrm{mg} / \mathrm{kg} .^{12}$ Despite dose adjustments, drug survival at 2 years is about $75 \%$, with rates of discontinuation due to treatment failures reported in up to $20 \%$ of the patients.

In contrast with infliximab, available data for adalimumab and etanercept, although more limited, suggest that dose intensification is not a common practice for patients with RA treated with those biological agents. If anything, data on the use of these agents suggest that a decrease of the dose of corticosteroids and/or methotrexate
(MTX) may be possible with time without compromising efficacy. ${ }^{18} 19$

Taking these data together, we conclude that a significant number of patients receiving infliximab require dose adjustment and that some patients may indeed be developing secondary failure and drug resistance. In the absence of prospective studies designed to examine dose adjustments and secondary failure, these data should be considered as preliminary and will require further validation. Because drug resistance is a relatively common event in the treatment of RA, we predict that drug resistance will be found with all three anti-TNF agents in common use today-albeit at different rates.

\section{WHAT IS THE BASIS FOR THE DIFFERENTIAL DRUG RESISTANCE OF ANTI-TNF AGENTS?}

Each one of the three anti-TNF agents is immunogenic to some extent.

Immunogenicity of anti-TNF agents has been assessed in different studies, but it is difficult to obtain direct comparisons owing to confounding factors such as concomitant drug treatment, assays used to determine immunogenicity, and study design. ${ }^{20}$ Initial studies for infliximab by Maini et al, showed that anti-infliximab antibodies were detected in $21 \%$ and $7 \%$ of patients with RA treated with 3, and $10 \mathrm{mg} / \mathrm{kg}$ infliximab, respectively, and were significantly lowered to $7 \%$ and $0 \%$ with concomitant low dose MTX. ${ }^{21 a}$ Subsequent studies have shown antiinfliximab antibodies in up to $11 \%$ of patients. ${ }^{1}$ Adalimumab appears to be less immunogenic than infliximab, with a cumulative incidence of antiadalimumab antibodies in $6 \%$ of patients with RA. ${ }^{20}$ The incidence was greater with lower doses, while concomitant MTX reduced the incidence to $1 \%$, which was about the incidence $(<1 \%)$ reported by Weinblatt et al in patients receiving concomitant MTX. $^{21}$ In controlled clinical trials of patients with rheumatoid arthritis treated with etanercept, $2 \%$ of patients became antibody positive. ${ }^{22}$

\section{"Antibodies to anti-TNF $\alpha$ agents are found during treatment, which may shorten the duration of clinical response" $^{\prime \prime}$}

Although the consequences of antibody formation against anti-TNF agents are not completely understood, recent data mainly from patients with Crohn's disease treated with infliximab suggest that such antibodies may be associated with an increased incidence of infusion reactions and a shortened duration of 
clinical response. ${ }^{23}$ Recently, in a small retrospective cohort study, Haraoui et al found higher anti-infliximab antibody concentrations in patients who needed dose escalation to achieve or maintain clinical responses, in conjunction with lower serum trough levels of infliximab. These results suggest that development of anti-infliximab antibodies may reduce the clinical efficacy of infliximab in some patients with RA. ${ }^{24}$ Whether the presence of these antibodies will assist in identifying patients more likely to have transfusion reactions or decreased efficacy remains to be seen. Of note, there are no data to suggest that antibodies induced to infliximab may cross react with other currently available anti-TNF $\alpha$ agents ${ }^{25}$; thus physicians may choose to switch from one to another anti-TNF agent.

\section{CONCLUSIONS}

Data provided by this and other LOS suggest that among $\mathrm{TNF} \alpha$ inhibitors, infliximab is more likely than other agents to require adjustment of the initial dose and frequency of administration, and that drug resistance may be developing in a subset of these patients The relative significance of this phenomenon in affecting drug effectiveness and the cost of treatment needs to be further clarified. Moreover, drug discontinuation rates, which are an important indirect measure of clinical effectiveness, were similar among the three agents, suggesting that any potential differences do not affect decisions about discontinuation of treatment. Whether with longer follow up of agents like adalimumab and etanercept, drug resistance will also become apparent, remains to be seen. Decrease of the dose of anti-TNF $\alpha$ agents required has not been consistently documented with any of the available agents in patients with established disease. Failure to gradually taper the dose of anti-TNF $\alpha$ agents in the face of a better control of disease activity and reduction of the "inflammatory burden", poses the question of whether some type of resistance to these agents is gradually developing.

In the meantime, physicians using infliximab (and to a lesser degree the other agents) should be aware of these observations, monitor disease activity closely, and adjust the dose of the drug as needed. At this point it is unlikely that drug resistance would affect significantly the initial choice of agent used to treat RA. Irrespective of the biological agent or the DMARD used, intensive outpatient monitoring of RA substantially improves disease activity, radiographic disease progression, physical function, and quality of life, highlighting the importance of tight control of disease activity with frequent dose adjustments. ${ }^{10}$

Ann Rheum Dis 2006;65:701-703. doi: $10.1136 /$ ard.2005.049890

\section{Authors' affiliations}

P I Sidiropoulos, D T Boumpas, Departments of Internal Medicine and Rheumatology, Clinical Immunology and Allergy, University of Crete, University Hospital, Voutes 71110 Heraklion, Greece

Correspondence to: Professor D T Boumpas, boumpasd@med.voc.gr

\section{REFERENCES}

1 Lipsky PE, van der Heijde DM, St Clair EW, Furst DE, Breedveld FC, Kalden JR, et al. Infliximab and methotrexate in the treatment of rheumatoid arthritis. Anti-Tumor Necrosis Factor Trial in Rheumatoid Arthritis with Concomitant Therapy Study Group. N Engl J Med 2000;343:1594-602.

2 Klareskog L, van der Heijde D, de Jager JP, Gough A, Kalden J, Malaise M, et al. Therapeutic effect of the combination of etanercept and methotrexate compared with each treatment alone in patients with rheumatoid arthritis: double-blind randomised controlled trial. Lancet 2004;363:675-81.

3 Keystone EC, Kavanaugh AF, Sharp JT, Tannenbaum H, Hua Y, Teoh LS, et al. Radiographic, clinical, and functional outcomes of treatment with adalimumab (a human anti-tumor necrosis factor monoclonal antibody) in patients with active rheumatoid arthritis receiving concomitant methotrexate therapy: a randomized, placebo-controlled, 52-week trial. Arthritis Rheum 2004;50:1400-11.

4 Sidiropoulos P, Bertsias G, Kritikos HD, Kouroumali H, Voudouris K, Boumpas DT. Infliximab treatment for rheumatoid arthritis, with dose titration based on the Disease Activity Score: dose adjustments are common but not always sufficient to assure sustained benefit. Ann Rheum Dis 2004;63:144-8

5 Finckh A, Simard JF, Gabay C, Guerne P-A, for the SCQM physicians. Evidence for differential acquired drug resistance to anti-tumour necrosis factor agents in rheumatoid arthritis. Ann Rheum Dis 2006;65:746-52.

6 Kristensen LE, Saxne T, Geborek P. The LUNDEX a new index of drug efficacy in clinical practice: results of a five-year observational study of treatment with infliximab and etanercept among rheumatoid arthritis patients in southern Sweden. Arthritis Rheum 2006;54:600-6.

7 Zink A, Listing J, Kary S, Ramlau P, StoyanovaScholz M, Babinsky K, et al. Treatment continuation in patients receiving biological agents or conventional DMARD therapy. Ann Rheum Dis 2005;64:1274-9.

8 Finckh A, Simard JF, Duryea J, Liang MH, Huang J, Daneel S, et al. The effectiveness of antitumor necrosis factor therapy in preventing progressive radiographic joint damage in rheumatoid arthritis: a population-based study. Arthritis Rheum 2006;54:54-9.

9 Wolfe F. Critical issues in longitudinal and observational studies: purpose, short versus long term, selection of study instruments, methods, outcomes, and biases. J Rheumatol 1999;26:469-72
10 Grigor C, Capell H, Stirling A, McMahon AD, Lock $\mathrm{P}$, Vallance $\mathrm{R}$, et al. Effect of a treatment strategy of tight control for rheumatoid arthritis (the TICORA study): a single-blind randomised controlled trial. Lancet 2004;364:263-9.

11 Oerlemans R, van der Heijden J, Vink J, Dijkmans BA, Kaspers GJ, Lems WF, et al. Acquired resistance to chloroquine in human CEM T cells is mediated by multidrug resistanceassociated protein 1 and provokes high levels of cross-resistance to glucocorticoids. Arthritis Rheum 2006:54:557-68.

12 Stern R, Wolfe F. Infliximab dose and clinical status: results of 2 studies in 1642 patients with rheumatoid arthritis. J Rheumatol 2004;31:1538-45.

13 van Vollenhoven R, Klareskog L. Dosage and frequency of infliximab in clinical practice: data from the STURE Registry [abstract]. Arthritis Rheum 2005;52(suppl):FRI0015.

14 van Vollenhoven $R$, Bratt J, Klareskog $\mathrm{L}$. Secondary loss of efficacy with TNF $\alpha$-antagonists. Data from the STURE Registry [abstract]. Arthritis Rheum 2005;52(suppl):275

15 van Vollenhoven RF, Klareskog L. Is there a longterm loss of efficacy with infliximab? Data from the STURE registry [abstract]. Ann Rheum Dis 2004;63(suppl):FRI0169.

16 St Clair EW, Wagner CL, Fasanmade AA, Wang B, Schaible T, Kavanaugh A, et al. The relationship of serum infliximab concentrations to clinical improvement in rheumatoid arthritis: results from ATTRACT, a multicenter, randomized, double-blind, placebo-controlled trial. Arthritis Rheum 2002;46:1451-9.

17 Nestorov I. Clinical pharmacokinetics of TNF antagonists: how do they differ? Semin Arthritis Rheum 2005;34(suppl 1):12-18.

18 Kremer JM, Weinblatt ME, Bankhurst AD, Bulpitt KJ, Fleischmann RM, Jackson CG, et al. Etanercept added to background methotrexate therapy in patients with rheumatoid arthritis: continued observations. Arthritis Rheum 2003:48: 1493-9.

19 Weinblatt ME, Keystone EC, Furst DE Kavanaugh AF, Chartash EK, Segurado OG Long-term efficacy and safety of adalimumab plus methotrexate in patients with rheumatoid arthritis: ARMADA 4-year extended study. Ann Rheum Dis 2006;65:753-9.

20 Anderson PJ. Tumor necrosis factor inhibitors: clinical implications of their different immunogenicity profiles. Semin Arthritis Rheum 2005;34(suppl 1):19-22.

21 Weinblatt ME, Keystone EC, Furst DE Moreland LW, Weisman MH, Birbara CA, et al. Adalimumab, a fully human anti-tumor necrosis factor alpha monoclonal antibody, for the treatment of rheumatoid arthritis in patients taking concomitant methotrexate: the ARMADA trial. Arthritis Rheum 2003;48:35-45.

21 a Maini RN, Breedveld FC, Kalden JR, Smolen JS, Davis D, Macfarlane JD, et al. Therapeutic efficacy of multiple intravenous infusions of antitumor necrosis factor alpha monoclonal antibody combined with low-dose weekly methotrexate in rheumatoid arthritis. Arthritis Rheum 1998:41:1552-63.

22 Foerder C, Rogge M. Immunogenicity of Enbrel: clinical trial observations [abstract]. Ann Rheum Dis 2002;61(suppl I):THU0032.

23 Baert F, Noman M, Vermeire S, Van Assche G, $D^{\prime}$ Haens G, Carbonez A, et al. Influence of immunogenicity on the long-term efficacy of infliximab in Crohn's disease. N Engl J Med 2003;348:601-8.

24 Haraoui B, Cameron L, Ouellet M, White B. Antiinfliximab antibodies in patients with rheumatoid arthritis who require higher doses of infliximab to achieve or maintain a clinical response. $J$ Rheumatol 2006;33:31-6.

25 Wagner CL, Schantz A, Barnathan E, Olson A Mascelli MA, Ford J, et al. Consequences of immunogenicity to the therapeutic monoclonal antibodies ReoPro and Remicade. Dev Biol (Basel) 2003; 1 12:37-53. 